\title{
Bromotyrosine-derived alkaloids from the Caribbean sponge Aplysina lacunosa
}

\author{
Qun Göthel, Thanchanok Sirirak and Matthias Köck
}

\author{
Full Research Paper \\ Address: \\ Alfred-Wegener-Institut, Helmholtz-Zentrum für Polar- und \\ Meeresforschung, Am Handelshafen 12, 27570 Bremerhaven, \\ Germany \\ Email: \\ Matthias Köck - mkoeck@awi.de \\ * Corresponding author \\ Keywords: \\ alkaloids; Aplysina lacunosa; bromotyrosine; marine natural products; \\ NMR spectroscopy \\ Beilstein J. Org. Chem. 2015, 11, 2334-2342. \\ doi:10.3762/bjoc.11.254 \\ Received: 25 March 2015 \\ Accepted: 01 September 2015 \\ Published: 26 November 2015 \\ Associate Editor: A. Kirschning \\ (C) 2015 Göthel et al; licensee Beilstein-Institut. \\ License and terms: see end of document.
}

Open Access

\begin{abstract}
Three new bromotyrosine-derived alkaloids 14-debromo-11-deoxyfistularin-3 (1), aplysinin A (2), and aplysinin B (3), together with 15 known compounds (4-18) were isolated from the sponge Aplysina lacunosa collected from Stirrup Cay, Bahamas. The structures of the isolated compounds were identified on the basis of MS and NMR data analysis. The ${ }^{13} \mathrm{C}$ NMR assignment of spirocyclohexadienylisoxazoline moieties of $\mathbf{1}$ and $\mathbf{2}$ were confirmed by an 1,1-ADEQUATE experiment. Compounds $\mathbf{1}$ and $\mathbf{2}$ showed a mild to moderate cytotoxic activities against KB-31 and FS4-LTM cell lines. Only aplysinin A (2) exhibited cytotoxicity against MCF-7 cells.
\end{abstract}

\section{Introduction}

Bromotyrosine-derived alkaloids are unique brominated metabolites which were isolated mainly from marine sponges of the order Verongida. For more than 50 years, bromotyrosine alkaloids raised the interests of synthetic and natural products chemists due to their high chemical diversity (besides the common spirocyclohexadienylisoxazoline moiety) and interesting biological activities. Since the first derivative 2,6-dibromo4-acetamido-4-hydroxycyclohexadienone was isolated in 1967 [1], a series of bromotyrosine alkaloids were discovered with various biological activities, including antiviral [2], antibiotic [3-5], $\mathrm{Na}^{+} / \mathrm{K}^{+}$ATPase inhibition [6-8], anti-HIV [9,10], antifungal [11], histidine-H3 antagonist [12], cytotoxic [13,14], and antimalarial activities [15-17]. During our investigation of the chemical constituents of Aplysina lacunosa (Aplysinidae, Verongida), three new bromotyrosine-derived alkaloids: 14-debromo-11-deoxyfistularin-3 (1), aplysinin A (2), and aplysinin B (3) (Figure 1), together with 15 known compounds were obtained. In this report we describe the structure elucidation of $\mathbf{1}$ to $\mathbf{3}$ and the biological activities of all the isolated compounds.

\section{Results and Discussion}

The freeze-dried sponge was extracted three times with $\mathrm{CH}_{2} \mathrm{Cl}_{2} / \mathrm{MeOH}(1: 1, \mathrm{v} / \mathrm{v})$. The resulting crude extract was parti- 


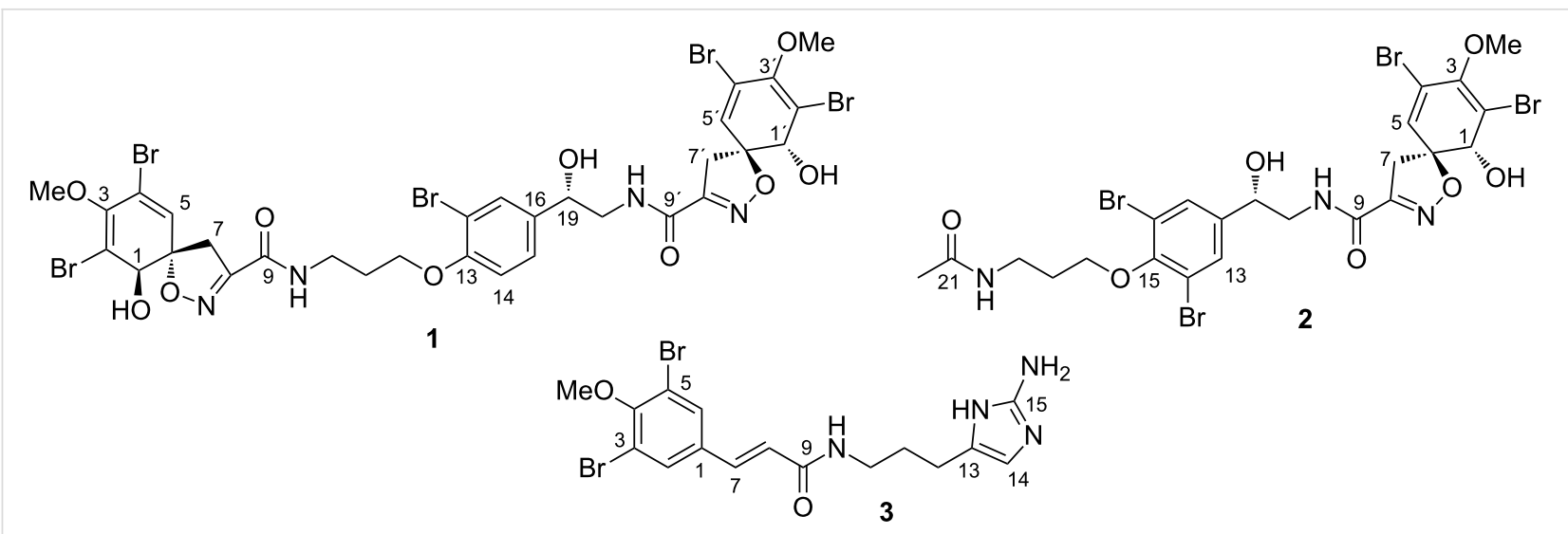

Figure 1: Three new bromotyrosine derivatives isolated from sponge Aplysina lacunosa: 14-debromo-11-deoxyfistularin-3 (1), aplysinin A (2), and aplysinin B (3).

tioned between $n$-hexane and $\mathrm{MeOH}$. The $\mathrm{MeOH}$ extract was further partitioned between ethyl acetate and $\mathrm{H}_{2} \mathrm{O}$. The resulting ethyl acetate phase was purified by vacuum liquid chromatography using silica gel with a stepwise gradient eluent from 100:0 to 80:20 $\left(\mathrm{CH}_{2} \mathrm{Cl}_{2} / \mathrm{MeOH}, \mathrm{v} / \mathrm{v}\right)$. The two fractions eluting with 97:3 and 94:6 $\left(\mathrm{CH}_{2} \mathrm{Cl}_{2} / \mathrm{MeOH}\right.$, v/v) were further purified by HPLC and yielded the three new bromotyrosine derivatives 14-debromo-11-deoxyfistularin-3 (1), aplysinins A (2) and B (3), as well as the 15 known compounds: 14-debromoaraplysillin I (4) [18], fistularin-3 (5) [19], 11,19-dideoxyfistularin-3 (6) [20], 19-deoxyfistularin-3 (7) [21], 11-deoxyfistularin-3 (8) [22], 11-ketofistularin-3 (9) [2], hexadellin B (10) [23], aerothionin (11) [13,24,25], 11-hydroxyaerothionin (12) [20], 11-oxoaerothionin (13) [13], 11-oxo-12-hydroxyaerothionin (14) [26], $N$-methyl-aerophobin-2 (15) [27], aeroplysinin-2 (16) [28], subereaphenol B (17) [29], and the unnamed bromotyrosine 18 [30]. Compounds 4 to 18 were identified by comparison of their MS data as well as ${ }^{1} \mathrm{H}$ and ${ }^{13} \mathrm{C}$ NMR chemical shifts with those reported in the literature (Figure 2).

Compound 1 was obtained as a white solid. The MS-ESI $(+)$ showed a characteristic pentabrominated ion peak cluster at $\mathrm{m} / \mathrm{z}$ 1037/1039/1041/1043/1045/1047 [M+ Na $]^{+}(1: 5: 10: 10: 5: 1)$. The molecular formula of $\mathrm{C}_{31} \mathrm{H}_{31} \mathrm{Br}_{5} \mathrm{~N}_{4} \mathrm{O}_{10}$ was deduced from HRMS-ESI(+) at $\mathrm{m} / z 1036.7844[\mathrm{M}+\mathrm{Na}]^{+}$(calcd for $\left.\mathrm{C}_{31} \mathrm{H}_{31}{ }^{79} \mathrm{Br}_{5} \mathrm{~N}_{4} \mathrm{O}_{10} \mathrm{Na}, 1036.7855\right)$ which required 16 double bond equivalents (DBEs). The ${ }^{13} \mathrm{C}$ NMR spectrum of 1 indicated two amide groups at $\delta 158.9\left(\mathrm{C}^{-} 9^{\prime}\right)$ and 159.5 (C-9), 14 olefinic carbons, two hetero-olefinic carbons at $\delta 154.5$ $\left(\mathrm{C}-8^{\prime}\right)$ and 155.0 (C-8), and 5 ring systems to fulfill the DBEs. The comparison of the ${ }^{1} \mathrm{H}$ and ${ }^{13} \mathrm{C}$ NMR data from positions C-1, C-1' to C-9, C-9' (Table 1) of $\mathbf{1}$ with those of aerothionin (11) $[13,24,25]$ allowed the assignment of the two dibromospirocyclohexadienylisoxazole carbonyl groups which was further confirmed by ${ }^{1} \mathrm{H},{ }^{13} \mathrm{C}$-HMBC and 1,1-ADEQUATE experiments. The ${ }^{13} \mathrm{C}$ NMR assignment of $\mathrm{C}-2$ and $\mathrm{C}-4$ were reversed before Ciminiello's revision in 1994 [26]. Nevertheless, the wrong assignment has still continued to be used as reference in the literature [29,31]. We therefore applied an 1,1ADEQUATE experiment which allows the selective observation of two-bond $\mathrm{H}, \mathrm{C}$ correlations [32]. The signals in the 1,1ADEQUATE spectrum from $\delta_{\mathrm{H}} 3.92\left(\mathrm{H}-1, \mathrm{H}-1^{\prime}\right)$ to $\delta_{\mathrm{C}} 113.6$ $\left(\mathrm{C}-2, \mathrm{C}-2^{\prime}\right)$ and from $\delta_{\mathrm{H}} 6.57\left(\mathrm{H}-5, \mathrm{H}-5^{\prime}\right)$ to $\delta_{\mathrm{C}} 120.9$ and 120.8 (C-4 and C-4', respectively) confirmed the assignments of $\mathrm{C}-2$, C-2' and C-4, C-4' from 1994 (Figure 3). ${ }^{1} \mathrm{H},{ }^{1} \mathrm{H}-\mathrm{COSY}$ correlations were observed among 9-NH $(\delta 8.60) / \mathrm{H}-10(\delta 3.36,2 \mathrm{H}) / \mathrm{H}-$ $11(\delta 1.95,2 \mathrm{H}) / \mathrm{H}-12(\delta 4.06,2 \mathrm{H})$ establishing an propanamine moiety. In a similar manner, ${ }^{1} \mathrm{H},{ }^{1} \mathrm{H}-\mathrm{COSY}$ correlations among $9^{\prime}-\mathrm{NH}(\delta 8.35) / \mathrm{H}-20(\delta 3.35) / \mathrm{H}-19(\delta 4.65,2 \mathrm{H})$ revealed the presence of a hydroxylethylamine moiety. The remaining signals at $\delta_{\mathrm{H}} 7.05(\mathrm{~d}, J=8.6 \mathrm{~Hz}, \mathrm{H}-14), 7.28(\mathrm{dd}, J=1.5$, $8.6 \mathrm{~Hz}, \mathrm{H}-15)$, and $7.52(\mathrm{~d}, J=1.5 \mathrm{~Hz}, \mathrm{H}-17)$ together with ${ }^{1} \mathrm{H},{ }^{13} \mathrm{C}-\mathrm{HMBC}$ correlations indicated the presence of a $1,2,4-$ trisubstituted phenoxy group. The phenoxy group was connected to the propanamine and the hydroxyethylamine substructures according to ${ }^{1} \mathrm{H},{ }^{13} \mathrm{C}-\mathrm{HMBC}$ correlations from $\mathrm{H}-12$ to $\delta_{\mathrm{C}} 153.8$ (C-13) and from $\mathrm{H}-19$ to $\delta_{\mathrm{C}} 137.2$ (C-16). Both sides of the linear fragment were connected to dibromospirocyclohexadiene moieties through amide bonds according to ${ }^{1} \mathrm{H},{ }^{13} \mathrm{C}-\mathrm{HMBC}$ correlations from $\mathrm{H}-7(\delta 3.21,3.63)$ and $9-\mathrm{NH}$ to $\mathrm{C}-9(\delta 159.1)$ as well as from H-7' $(\delta 3.19,3.62)$ and $9^{\prime}-\mathrm{NH}$ to $\mathrm{C}-9^{\prime}(\delta 159.0)$. The structure of $\mathbf{1}$ is closely related to 11-deoxyfistularin-3 (8) which was originally isolated from the Caribbean sponge Aplysina fistularis insularis [22]. The only difference between $\mathbf{1}$ and $\mathbf{8}$ is the lack of one bromine atom in the central benzene ring of compound 1 at C-14. Therefore, compound 1 was named 14-debromo-11-deoxyfistularin-3. The ${ }^{13} \mathrm{C}$ chemical shifts assignment of $\mathbf{1}$ according to the $1,1-$ ADEQUATE suggested a revision of the chemical shifts of C-2, 
<smiles>COC1=C(Br)C(O)[C@]2(C=C1Br)CC(C(=O)NCCCOc1ccc(CCN)cc1Br)=NO2</smiles>

4<smiles>COC1=C(Br)[C@H](O)[C@@]2(C=C(Br)C(Br)=C[C@H]2O)C1</smiles><smiles>[R]C(CNC(=O)C1=NO[C@]2(C=C(Br)C(OC)=C(Br)[C@@H]2O)C1)c1cc(Br)c(OCC([R])([R])CNC(=O)C2=NO[C@]3(C=C(Br)C(OC)=C(Br)[C@H]3O)C2)c(Br)c1</smiles><smiles>[R]C(CNC(=O)C1=NO[C@]2(C=C(Br)C(OC)=C(Br)[C@@H]2O)C1)C([R])([R])CNC(=O)C1=NO[C@]2(C=C(Br)C(OC)=C(Br)[C@@H]2O)C1</smiles>

$5: \mathrm{R}^{1}=\mathrm{R}^{3}=\mathrm{OH}, \mathrm{R}^{2}=\mathrm{H}$

$6: \mathrm{R}^{1}=\mathrm{R}^{2}=\mathrm{R}^{3}=\mathrm{H}$

$7: \mathrm{R}^{1}=\mathrm{OH}, \mathrm{R}^{2}=\mathrm{R}^{3}=\mathrm{H}$

$8: R^{1}=R^{2}=H, R^{3}=\mathrm{OH}$<smiles></smiles>

15<smiles>COC1=C(Br)[C@H]2OC(=O)C[C@]2(O)C=C1Br</smiles>

16<smiles>COC(=O)Cc1cc(Br)c(O)c(Br)c1O</smiles>

17

$$
\begin{aligned}
& 11: R^{1}=R^{2}=R^{3}=H \\
& 12: R^{1}=O H, R^{2}=R^{3}=H \\
& 13: R^{1}=R^{2}=O, R^{3}=H \\
& 14: R^{1}=R^{2}=O, R^{3}=O H
\end{aligned}
$$<smiles>COC(=O)C1=NO[C@]2(C=C(Br)C(OC)=C(Br)[C@@H]2O)C1</smiles>

18

Figure 2: Bromotyrosine alkaloids and brominated compounds isolated from the sponge Aplysina lacunosa.

C-2', C-6, and C-6' of the two related compounds 11-deoxyfistularin-3 (8) and 14-debromoaraplysilin I (4) [18] (Table 1 and Table 2, respectively).

The relative configuration of the spiroisoxazoline rings of $\mathbf{1}$ was investigated using a NOESY experiment. NOEs were observed between $\delta_{\mathrm{H}} 6.36(1-\mathrm{OH})$ and $3.60(\mathrm{H}-7) ; 6.37\left(1^{\prime}-\mathrm{OH}\right)$ and 3.63 (H-7'); 3.57 (2H, H-5-and H-5') and 3.21 (2H, H-7 and $\left.\mathrm{H}-7^{\prime}\right)$ indicated a trans-hydroxyspiroisoxazoline ring which was supported by a W-coupling between the olefinic proton $\mathrm{H}-5$ and the methine proton $\mathrm{H}-1\left({ }^{4} \mathrm{~J} \sim 0.7 \mathrm{~Hz}\right)[28,33]$. An NOE was also observed between $\delta_{\mathrm{H}} 5.54(19-\mathrm{OH})$ and $\delta_{\mathrm{H}} 6.37\left(1^{\prime}-\mathrm{OH}\right)$ suggested that both hydroxy groups are on the same side of the molecule. The absolute configuration of compound $\mathbf{1}$ was investigated by $C D$ spectroscopy. The CD spectrum of $\mathbf{1}$ showed positive Cotton effects $\left(\lambda_{\max } 248, \Delta \varepsilon+5.16\right.$ $\left.\lambda_{\max } 285, \Delta \varepsilon+4.58\right)$ with the same sign and magnitude as observed for $(+)$-aerothionin (11) $[25,34,35]$. Thus, the absolute configuration of spiroisoxazoline moieties were assigned as $1,1^{\prime}-(R), 6,6^{\prime}-(S)$ (Figure 1). The absolute configuration of C-19 was assigned as 19- $(R)$ according to NOE data which is in agreement with the proposed configuration by Molinski and co-workers $[34,36]$. The configuration of C-19 of fistularin-3 (5) was proposed to be the same as of 19, chemical fragmentation of 5 releasing from the sponge Aplysina spp. after induction by tissue damage (Figure 4). However, the conversion from 5 to $\mathbf{1 9}$ has not been confirmed. A single data set in the ${ }^{13} \mathrm{C}$ NMR spectrum supported the presence of one diastereomer of 1 .

Compound 2 was isolated as a white solid. MS-ESI $(+)$ data of 2 showed a pseudomolecular ion cluster at $\mathrm{m} / \mathrm{z}$ 793/795/797/799/ $801[\mathrm{M}+\mathrm{Na}]^{+}(1: 4: 6: 4: 1)$ indicating a tetrabrominated compound. HRMS-ESI(+) of 2 at $m / z 793.8320[\mathrm{M}+\mathrm{Na}]^{+}$ suggested a molecular formula of $\mathrm{C}_{23} \mathrm{H}_{25} \mathrm{Br}_{4} \mathrm{~N}_{3} \mathrm{O}_{7}$ (calcd for $\left.\mathrm{C}_{23} \mathrm{H}_{25}{ }^{79} \mathrm{Br}_{4} \mathrm{~N}_{3} \mathrm{O}_{7} \mathrm{Na}, 793.8324\right)$. The ${ }^{1} \mathrm{H}$ and ${ }^{13} \mathrm{C}$ NMR spectra of $\mathbf{2}$ resemble to $\mathbf{1}$ except that the spectrum of $\mathbf{2}$ showed one extra methyl group $\left(\delta_{\mathrm{H}} 1.80 \mathrm{~s} ; \delta_{\mathrm{C}} 22.6\right)$ and one aromatic proton less $\left(\mathrm{C}-14, \delta_{\mathrm{H}} 7.05\right.$ for 1$)$. According to one set of $\delta_{\mathrm{H}} 3.17$ (H-7), 3.61 (H-7), 3.93 (H-1), 6.56 (H-5), $\delta_{\mathrm{C}} 39.7$ (C-7), 73.5 


\begin{tabular}{|c|c|c|c|c|c|c|}
\hline \multirow{2}{*}{ position } & \multicolumn{3}{|c|}{1} & \multicolumn{2}{|c|}{8} & \multirow{2}{*}{$\frac{\mathbf{8}^{a}}{\delta_{C}}$} \\
\hline & $\delta_{C}$ & $\delta_{H}$ & 1,1-ADEQ & $\delta_{C}$ & $\delta_{H}$ & \\
\hline $1,1^{\prime}$ & 73.6, CH; 73.5, CH & $3.92,2 \mathrm{H} \mathrm{s}$ & $2,2^{\prime}, 6,6^{\prime}$ & $74.1 ; 74.0$ & $3.93, \mathrm{~d}(7.9)$ & $74.67 ; 74.60$ \\
\hline $2,2^{\prime}$ & $113.6,2 \mathrm{C}$ & - & - & 113.6 & - & $121.66^{b}$ \\
\hline $3,3^{\prime}$ & $147.1,2 \mathrm{C}$ & - & - & 147.6 & - & 147.92 \\
\hline $4,4^{\prime}$ & $120.9, \mathrm{C} ; 120.8, \mathrm{C}$ & - & - & $121.4 ; 121.3$ & - & $115.16^{\mathrm{b}}$ \\
\hline $5,5^{\prime}$ & $\begin{array}{l}\text { 131.2, } \mathrm{CH} ; 131.1 \\
\mathrm{CH}\end{array}$ & $6.57,2 \mathrm{H} \mathrm{s}$ & $6,6^{\prime}$ & $131.7 ; 131.6$ & $\begin{array}{l}6.57, \mathrm{~s} \\
6.59, \mathrm{~s}\end{array}$ & $132.31 ; 132.15$ \\
\hline $6,6^{\prime}$ & $90.3, \mathrm{C} ; 90.2, \mathrm{C}$ & - & - & $90.8 ; 90.7$ & - & $91.78 ; 91.72$ \\
\hline \multirow[t]{2}{*}{7} & $40.0^{\mathrm{c}}, \mathrm{CH}_{2}$ & $3.21, \mathrm{~d}(18.2)$ & 6,8 & 39.9 & $3.22, \mathrm{~d}(18.2)$ & 40.27 \\
\hline & & $3.63, \mathrm{~d}(18.2)$ & & & $3.63, \mathrm{~d}(18.2)$ & \\
\hline $7^{\prime}$ & $39.9^{c}, \mathrm{CH}_{2}$ & $\begin{array}{l}3.19, \mathrm{~d}(18.2) \\
3.62, \mathrm{~d}(18.2)\end{array}$ & $6^{\prime}, 8^{\prime}$ & 39.6 & $\begin{array}{l}3.18, \mathrm{~d}(18.1) \\
3.62, \mathrm{~d}(18.1)\end{array}$ & \\
\hline $8,8^{\prime}$ & 154.5, C; 154.6, C & - & - & $155.0 ; 154.9$ & - & $155.23 ; 155.10$ \\
\hline $9,9^{\prime}$ & 159.1, C; 159.0, C & - & - & $159.5 ; 159.4$ & - & $160.44 ; 160.05$ \\
\hline 10 & $36.1, \mathrm{CH}_{2}$ & $3.36^{\mathrm{C}}, 2 \mathrm{H}$, overlapped & 11 & 36.7 & - & 37.13 \\
\hline 11 & $28.6, \mathrm{CH}_{2}$ & $1.95,2 \mathrm{H}$ qui $(6.4)$ & 10,12 & 29.9 & 2.01, qui (7.2) & 30.37 \\
\hline 12 & $66.5, \mathrm{CH}_{2}$ & $4.06,2 \mathrm{H} \mathrm{t}(6.2)$ & 11 & 71.7 & $3.98, \mathrm{t}(6.4)$ & 71.51 \\
\hline 13 & 153.8, C & - & - & 151.8 & - & 152.27 \\
\hline 14 & 113.3, $\mathrm{CH}$ & $7.05, \mathrm{~d}(8.6)$ & 13,15 & 117.8 & - & 118.35 \\
\hline 15 & 126.7, CH & $7.28, \mathrm{dd}(1.5,8.6)$ & - & 130.9 & $7.58, \mathrm{~s}$ & 130.90 \\
\hline 16 & 137.2, C & - & - & 143.1 & - & 143.35 \\
\hline 17 & $130.5, \mathrm{CH}$ & $7.52, \mathrm{~d}(1.5)$ & - & 130.9 & $7.58, \mathrm{~s}$ & 130.90 \\
\hline 18 & $110.8, \mathrm{C}$ & - & - & 117.8 & - & 118.35 \\
\hline 19 & $69.9, \mathrm{CH}$ & $4.65, \mathrm{dt}(4.5,7.2)$ & 16,20 & 69.9 & $4.69, \mathrm{q}(5.3)$ & 70.70 \\
\hline 20 & $46.8, \mathrm{CH}_{2}$ & $3.35^{c}, 2 \mathrm{H}$ overlapped & - & 46.8 & $\begin{array}{l}3.29, \mathrm{~m} \\
3.34^{\mathrm{c}}\end{array}$ & 47.99 \\
\hline 3, 3'-OMe & $59.7,2 \mathrm{CH}_{3}$ & $3.64,6 \mathrm{H} \mathrm{s}$ & - & 60.1 & $3.66, \mathrm{~s}$ & 59.75 \\
\hline 9-NH & - & $8.60, \mathrm{t}(5.8)$ & - & - & $8.57, \mathrm{t}(5.7)$ & - \\
\hline $9^{\prime}-\mathrm{NH}$ & - & $8.35, \mathrm{t}(5.8)$ & - & - & $8.39, \mathrm{t}(5.7)$ & - \\
\hline $1-\mathrm{OH}$ & - & $6.36^{d}$ & - & - & $6.36, \mathrm{~d}(7.9)$ & - \\
\hline $1^{\prime}-\mathrm{OH}$ & - & $6.37^{d}$ & - & - & $6.37, \mathrm{~d}(7.9)$ & - \\
\hline $19-\mathrm{OH}$ & - & $5.54, \mathrm{~d}(4.5)$ & - & - & $5.73, \mathrm{~d}(5.3)$ & - \\
\hline
\end{tabular}

Table 2: ${ }^{13} \mathrm{C}$ NMR data $\left(600 \mathrm{MHz}\right.$, DMSO- $d_{6}$ ) of the central benzene ring of the isolated compounds 1 and 4 .

\begin{tabular}{llll} 
position & $\mathbf{1}$ & $\mathbf{4}$ & $\mathbf{4}^{\mathrm{a}}$ \\
\hline 13 & 153.8 & 153.3 & 153.4 \\
14 & 113.3 & 111.3 & $134.4^{\mathrm{b}}$ \\
15 & 126.7 & 133.8 & 133.4 \\
16 & 137.2 & 133.4 & $112.2^{\mathrm{b}}$ \\
17 & 130.5 & 129.6 & 128.8 \\
18 & 110.8 & 113.6 & 113.3 \\
\hline
\end{tabular}

aThe ${ }^{13} \mathrm{C}$ NMR data were obtained in $\mathrm{CDCl}_{3}$ [18]. ${ }^{\text {b} A s s i g n m e n t s ~}$ should be reversed.
(C-1), 90.3 (C-6), 113.1 (C-2), 120.9 (C-4), 131.1 (C-5), 147.1 (C-3), 154.3 (C-8), and 159.0 (C-9) together with ${ }^{1} \mathrm{H},{ }^{13} \mathrm{C}-\mathrm{HMBC}$ correlations revealed that $\mathbf{2}$ consist of only one spirocyclohexadienylisoxazoline moiety in comparison with $\mathbf{1}$. The structure determination of $\mathbf{2}$ was accomplished based on ${ }^{1} \mathrm{H},{ }^{1} \mathrm{H}-\mathrm{COSY}$ and ${ }^{1} \mathrm{H},{ }^{13} \mathrm{C}-\mathrm{HMBC}$ correlations in the same manner as for 1 . Once again, ${ }^{1} \mathrm{H},{ }^{1} \mathrm{H}-\mathrm{COSY}$ revealed propanamine $[\mathrm{H}-18(\delta 3.96,2 \mathrm{H}) / \mathrm{H}-19(\delta \quad 1.91,2 \mathrm{H}) / \mathrm{H}-20$ $(\delta 3.25) / 20-\mathrm{NH}(\delta 7.86)]$ and hydroxyethylamine $[9-\mathrm{NH}(\delta$ $8.39) / \mathrm{H}-10(\delta 3.33,2 \mathrm{H}) / \mathrm{H}-11$ ( $\delta$ 4.67)/11-OH $(\delta$ 5.72)] substructures. The signals at $\delta_{\mathrm{H}} 7.57(2 \mathrm{H}, \mathrm{s}, \mathrm{H}-13,17), \delta_{\mathrm{C}} 117.3$ (C-14, 16), 130.4 (C-13, 17), 142.5 (C-12), and 151.4 (C-15) and ${ }^{1} \mathrm{H},{ }^{13} \mathrm{C}-\mathrm{HMBC}$ correlations among those signals showed a 1,2,4,6-dibromophenyl moiety. The substructures were assem- 


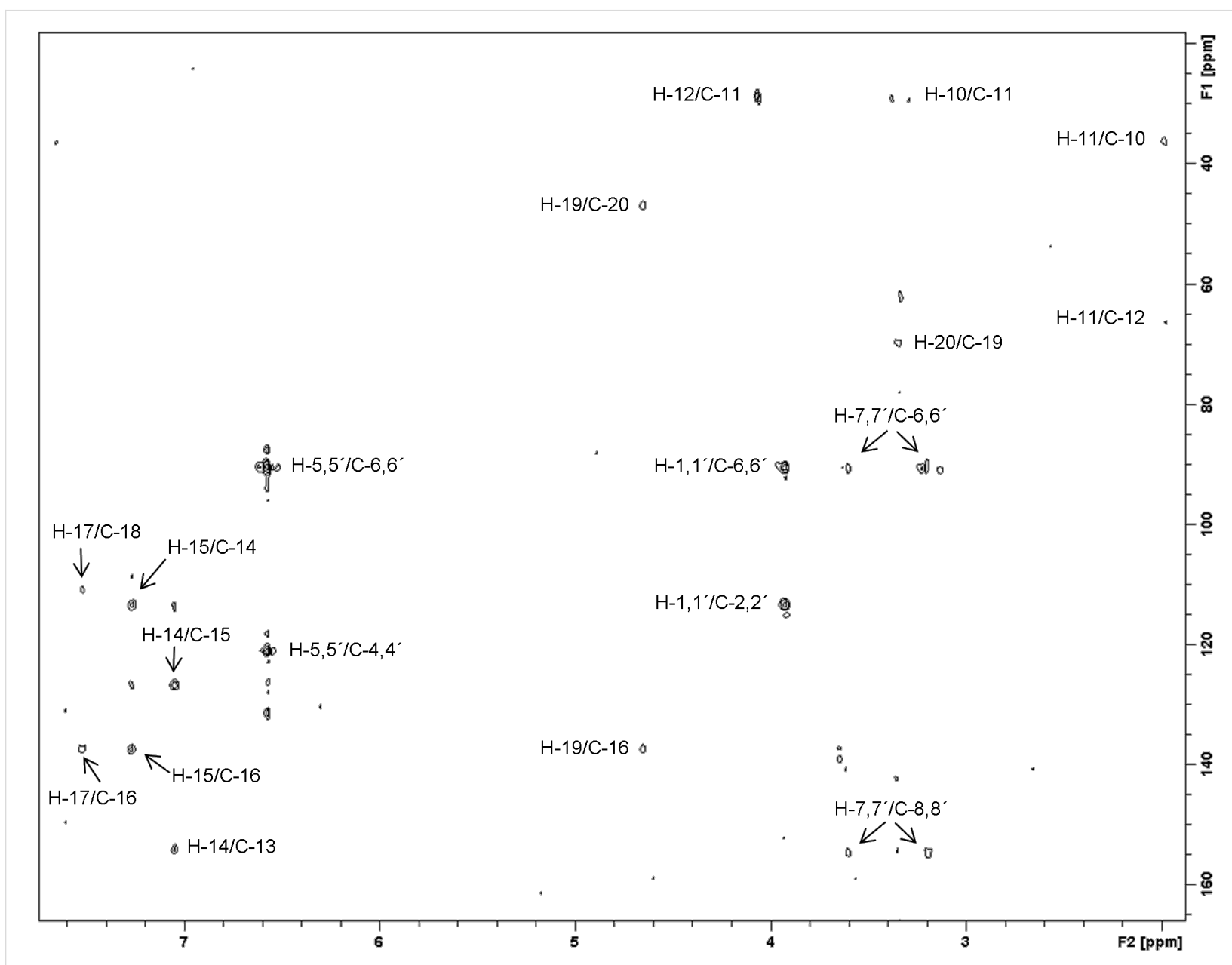

Figure 3: 1,1-ADEQUATE spectrum of 14-debromo-11-deoxyfistularin-3 (1).

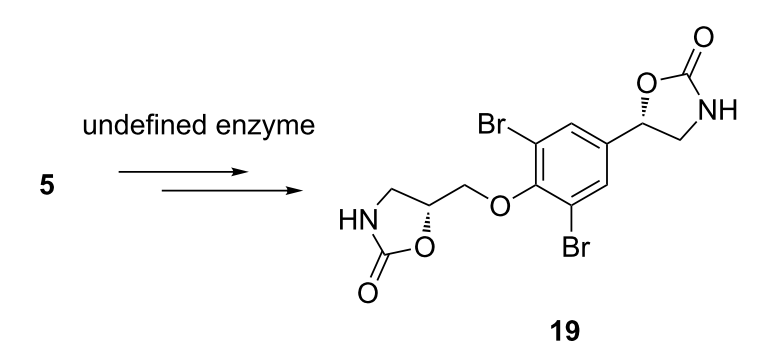

Figure 4: The tissue damage induced chemical conversion from fistularin-3 (5) to 19 by an undefined enzyme in the sponge Aplysina spp. [36].

bled by ${ }^{1} \mathrm{H},{ }^{13} \mathrm{C}-\mathrm{HMBC}$ correlations from $\mathrm{H}-10$ to $\mathrm{C}-9$ and $\mathrm{C}-12$, from $\mathrm{H}-11$ to $\mathrm{C}-12$ and $\mathrm{C}-13$ as well as from $\delta_{\mathrm{H}} \mathrm{H}-18$ to $\mathrm{C}-15$. The terminal of side chain was connected to an acetamide moiety according to ${ }^{1} \mathrm{H},{ }^{13} \mathrm{C}-\mathrm{HMBC}$ correlations from $\mathrm{H}-20$, $20-\mathrm{NH}$, and $\delta_{\mathrm{H}} 1.80(3 \mathrm{H} ; \mathrm{H}-22)$ to $\delta_{\mathrm{C}} 169.1$ (C-21). Compound $\mathbf{2}$ was named aplysinin A. The structure of $\mathbf{2}$ is similar to rightside portions of $\mathbf{1}$ and $\mathbf{8}$ (start at C-10). However, compound $\mathbf{2}$ contained an acetamide in the left-side portion instead of a ring system in comparison with $\mathbf{8}$ and showed great similarity with hexadellin B (10) isolating from the same organism. Hexadellin B (10) was originally isolated from the sponge Hexadella sp. The spectroscopic data of $\mathbf{1 0}$ was coincidently obtained from diacetylhexadellin B (20, Figure 5 and Table 3) [23] which supported the assignment of the acetamide moiety. The relative<smiles>COC1=C(Br)C(OC(C)=O)[C@]2(C=C1Br)C=C(C(=O)NCCc1cc(Br)c(OCCCNC(C)=O)c(Br)c1)C2</smiles>

20

Figure 5: Diacetylhexadellin B (20) isolated from sponge Hexadella sp. 


\begin{tabular}{|c|c|c|c|c|c|}
\hline \multirow{2}{*}{ position } & \multicolumn{2}{|r|}{2} & \multicolumn{2}{|c|}{10} & \multirow{2}{*}{$\begin{array}{l}\mathbf{2 0}^{\mathrm{a}} \\
\delta_{C}\end{array}$} \\
\hline & $\delta_{C}$ & $\delta_{H}$ & $\delta_{C}$ & $\delta_{H}$ & \\
\hline 1 & $73.5, \mathrm{CH}$ & $3.93, \mathrm{~d}(8.2)$ & 74.0 & $3.92, \mathrm{~d}(7.3)$ & 73.1 \\
\hline 2 & $113.1, \mathrm{C}$ & - & 113.5 & - & $122.1^{b}$ \\
\hline 3 & 147.1, C & - & 147.6 & - & 149.7 \\
\hline 4 & $120.9, \mathrm{C}$ & - & 121.4 & - & $107.8^{b}$ \\
\hline 5 & $131.1, \mathrm{CH}$ & $6.56, \mathrm{~s}$ & 131.6 & $6.58, d(0.8)$ & 130.2 \\
\hline 6 & $90.3, \mathrm{C}$ & - & 90.7 & - & 89.9 \\
\hline 7 & 39.7, $\mathrm{CH}_{2}$ & $\begin{array}{l}3.17, \mathrm{~d}(18.4) \\
3.61, \mathrm{~d}(18.4)\end{array}$ & 39.6 & $\begin{array}{l}3.19, \mathrm{~d}(18.1) \\
3.60, \mathrm{~d}(18.1)\end{array}$ & 39.9 \\
\hline 8 & $154.3, \mathrm{C}$ & - & 154.9 & - & 153.5 \\
\hline 9 & 159.0, C & - & 159.4 & - & 158.6 \\
\hline 10 & $46.3, \mathrm{CH}_{2}$ & $3.33,2 \mathrm{H}$, overlapped & 40.4 & $3.38, \mathrm{~m}$ & 40.4 \\
\hline 11 & $69.3, \mathrm{CH}$ & $4.67, \mathrm{t}(6.1)$ & 33.6 & $2.77, \mathrm{t}(7.1)$ & 34.4 \\
\hline 12 & $142.5, \mathrm{C}$ & - & 139.5 & - & 137.2 \\
\hline 13,17 & $130.4,2 \mathrm{CH}$ & $7.57,2 \mathrm{H}, \mathrm{s}$ & 133.5 & $7.54, \mathrm{~s}$ & 132.8 \\
\hline 14,16 & $117.3,2 \mathrm{C}$ & - & 117.7 & - & 118.2 \\
\hline 15 & $151.4, \mathrm{C}$ & - & 150.9 & - & 151.5 \\
\hline 18 & $71.3, \mathrm{CH}_{2}$ & $3.96,2 \mathrm{H}, \mathrm{t}(6.2)$ & 70.8 & $4.00, \mathrm{t}(6.1)$ & 72.1 \\
\hline 19 & 29.8, $\mathrm{CH}_{2}$ & $1.91,2 \mathrm{H}, \mathrm{q}(6.9)$ & 28.2 & $2.08, \mathrm{~m}$ & 29.4 \\
\hline 20 & $35.7, \mathrm{CH}_{2}$ & $3.25,2 \mathrm{H}, \mathrm{q}(6.7)$ & 37.0 & $3.08, \mathrm{br} \mathrm{s}$ & 37.7 \\
\hline 21 & $169.1, \mathrm{C}$ & - & - & - & 170.0 \\
\hline 22 & 22.6, $\mathrm{CH}_{3}$ & $1.80,3 \mathrm{H}, \mathrm{s}$ & - & - & 23.6 \\
\hline 3-OMe & $59.6, \mathrm{CH}_{3}$ & $3.65,3 \mathrm{H}, \mathrm{s}$ & 60.1 & $3.65, \mathrm{~s}$ & 60.3 \\
\hline $9-\mathrm{NH}$ & - & $8.39, \mathrm{t}(5.9)$ & - & $8.59, \mathrm{t}(5.9)$ & - \\
\hline $1-\mathrm{OH}$ & - & $6.36, d(8.2)$ & - & $6.37, \mathrm{~d}(7.8)$ & - \\
\hline $11-\mathrm{OH}$ & - & $5.72, \mathrm{~d}(4.4)$ & - & - & - \\
\hline 20-NH & - & $7.86, \mathrm{t}(5.3)$ & - & - & - \\
\hline
\end{tabular}

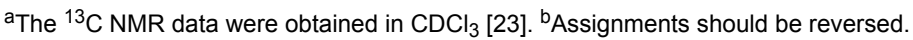

configuration of the spiroisoxazoline moiety (C-1 and C-6) was determined by comparison of the ${ }^{1} \mathrm{H}$ and ${ }^{13} \mathrm{C}$ NMR data with $\mathbf{1 0}$ and 20. The NOESY spectrum showed correlations between $\delta_{\mathrm{H}}$ $6.36(1-\mathrm{OH})$ and $3.61(\mathrm{C}-7)$ as well as between $6.56(\mathrm{H}-6)$ and 3.17 (H-7) suggesting a trans-hydroxyspiroisoxazoline ring similar to compound 1. An NOE was also observed between $\delta_{\mathrm{H}}$ $5.72(11-\mathrm{OH})$ and $1-\mathrm{OH}$ indicating the same planar alignment of both hydroxy groups. The absolute configuration of spiroisoxazoline moiety was confirmed as $1-(R), 6-(S)$ by positive Cotton effects $\left(\lambda_{\max } 252, \Delta \varepsilon+4.77, \lambda_{\max } 283, \Delta \varepsilon+3.34\right)$ comparing to $(+)$-aerothionin (11) in the same manner of $\mathbf{1}$ $[25,37]$. The arrangement of $1-\mathrm{OH}$ and $19-\mathrm{OH}$ on the same side of the structure allowed assigning the configuration of C-19. The presence of one diastereomer of 2 was confirmed by a single data set in the ${ }^{13} \mathrm{C}$ NMR spectrum.

Compound 3 was isolated together with $N$-methylaerophobin-2 (15) as a mixture (approximate ratio 1:5). The ESIMS spectrum exhibited a 1:2:1 ion cluster at $\mathrm{m} / \mathrm{z} 457 / 459 / 461$, indicating the presence of two bromine atoms. The HRMS-ESI $(+)$ spectrum revealed a pseudomolecular ion $[\mathrm{M}+\mathrm{H}]^{+}$at $\mathrm{m} / z$ 456.9892, which indicated a molecular formula of $\mathrm{C}_{16} \mathrm{H}_{18} \mathrm{Br}_{2} \mathrm{~N}_{4} \mathrm{O}_{2}$ (calcd for $\mathrm{C}_{16} \mathrm{H}_{19}{ }^{79} \mathrm{Br}_{2} \mathrm{~N}_{4} \mathrm{O}_{2}, 456.9875$ ), containing nine DBEs. Two singlet aromatic protons $\delta_{\mathrm{H}} 7.88\left(\delta_{\mathrm{C}} 131.7\right)$ suggested a tetrasubstituted benzene pattern which was confirmed by ${ }^{1} \mathrm{H},{ }^{13} \mathrm{C}-\mathrm{HMBC}$ correlations; from $\delta_{\mathrm{H}} 7.88(2 \mathrm{H}, \mathrm{H}-2, \mathrm{H}-6)$ to $\delta_{\mathrm{C}} 118.5(\mathrm{C}-3, \mathrm{C}-5), \delta_{\mathrm{C}} 154.4(\mathrm{C}-4), \delta_{\mathrm{C}} 131.7$ (C-2, C-6), and $\delta_{\mathrm{C}} 135.7(\mathrm{C}-7)$, from $\delta_{\mathrm{H}} 3.82$ (4-OMe) to C-4. The connection of the benzene fragment to the $E$-vinyl moiety was confirmed by HMBC correlations from two olefinic protons $\delta_{\mathrm{H}} 7.33$ (d, $J=15.8 \mathrm{~Hz}, \mathrm{H}-7)$ and $\delta_{\mathrm{H}} 6.66(\mathrm{~d}, J=15.8 \mathrm{~Hz}, \mathrm{H}-8)$ to $\delta_{\mathrm{C}} 134.9$ $(\mathrm{C}-1) .{ }^{1} \mathrm{H},{ }^{1} \mathrm{H}-\mathrm{COSY}$ correlations in between $9-\mathrm{NH}(\delta 8.17) /$ $\mathrm{H}-10(\delta 3.20,2 \mathrm{H}) / \mathrm{H}-11(\delta 1.71,2 \mathrm{H}) / \mathrm{H}-12(\delta 2.45,2 \mathrm{H})$ indicated a propanamine fragment which was connected to a 2-aminoimidazole moiety according to ${ }^{1} \mathrm{H},{ }^{13} \mathrm{C}-\mathrm{HMBC}$ correlations from $\mathrm{H}-12$ to $\delta_{\mathrm{C}} 126.8$ (C-13) and 109.2 (C-14) (Table 4). The two substructures are connected through an amide bond according to the ${ }^{1} \mathrm{H},{ }^{13} \mathrm{C}-\mathrm{HMBC}$ correlations from $\mathrm{H}-7,9-\mathrm{NH}$, 
and $\mathrm{H}-10$ to $\delta_{\mathrm{C}} 165.0(\mathrm{C}-9)$. The structure of 3 is very similar to compound 21 (Figure 6) which was isolated from the Caribbean sponge Verongula sp. [38]. In comparison with 21, compound 3 showed one extra aliphatic carbon and was named aplysinin B.

\begin{tabular}{|c|c|c|c|}
\hline \multirow{2}{*}{ position } & \multicolumn{2}{|c|}{3} & \multirow{2}{*}{$\frac{\mathbf{2 1}^{a}}{\delta_{C}}$} \\
\hline & $\delta_{C}$ & $\delta_{\mathrm{H}}$ & \\
\hline 1 & $134.9, \mathrm{C}$ & - & 136.36 \\
\hline 2,6 & 131.7, $\mathrm{CH}$ & $7.88,2 \mathrm{H}, \mathrm{s}$ & 132.98 \\
\hline 3,5 & $118.5, \mathrm{C}$ & - & 119.48 \\
\hline 4 & $154.4, \mathrm{C}$ & - & 154.46 \\
\hline 7 & $135.7, \mathrm{CH}$ & $7.33, \mathrm{~d}(15.8)$ & 138.42 \\
\hline 8 & $124.9, \mathrm{CH}$ & $6.66, d(15.8)$ & 123.95 \\
\hline 9 & 165.0, C & - & 167.50 \\
\hline 10 & $38.4, \mathrm{CH}_{2}$ & $3.20,2 \mathrm{H}, \mathrm{m}$ & 39.27 \\
\hline 11 & 28.1, $\mathrm{CH}_{2}$ & $1.71,2 \mathrm{H}, \mathrm{m}$ & 25.92 \\
\hline 12 & $22.0, \mathrm{CH}_{2}$ & $2.45,2 \mathrm{H}, \mathrm{m}$ & 125.89 \\
\hline 13 & $126.8, \mathrm{C}$ & - & 110.87 \\
\hline 14 & $109.2, \mathrm{CH}$ & $6.60, s$ & 146.43 \\
\hline 15 & $147.4, \mathrm{C}$ & - & - \\
\hline 4-OMe & $61.0, \mathrm{CH}_{3}$ & $3.82, \mathrm{~s}$ & 61.26 \\
\hline 9-NH & - & $8.17, \mathrm{t}(5.58)$ & - \\
\hline
\end{tabular}

${ }^{\text {a The }}{ }^{13} \mathrm{C}$ NMR data were obtained in $\mathrm{CD}_{3} \mathrm{OD}$ [38].<smiles>CCCNC(=O)/C=C/c1cc(Br)c(OC)c(Br)c1</smiles>

Figure 6: Bromotyrosine alkaloid (21) isolated from the sponge Verongula sp.

The new compounds 14-debromo-11-deoxyfistularin-3 (1) and aplysinin A (2) were tested for their antimicrobial activity against different Gram-positive and Gram-negative bacteria, fungi, and for their antiproliferative activity. Aplysinin B (3) was not subjected to any biological activity test due to the minute amount and its existence as the minor compound of a mixture. The results showed that $\mathbf{1}$ and $\mathbf{2}$ exhibited mild cytotoxic activity against KB-31 epidermoid carcinoma cells $\left(\mathrm{IC}_{50}=69\right.$ and $26 \mu \mathrm{M}$, respectively). Only 2 showed mild toxicity against the breast cancer cell line MCF-7 and to FS4-LTM conditional immortalization human fibroblasts $\left(\mathrm{IC}_{50}=78\right.$ and $32 \mu \mathrm{M}$, respectively). The cytotoxicities of the known compounds (4-17) are also listed in Table 5. None of the isolated compounds showed any antimicrobial activity.

\begin{tabular}{|lllll}
\hline \multicolumn{5}{|c}{ Table 5: Cytotoxicity of the isolated compounds $\left(\mathrm{IC}_{50}\right){ }^{\text {a }}$} \\
compound & \multicolumn{5}{c}{$\mathrm{IC}_{50}[\mu \mathrm{M}]$} \\
& L929 & KB-31 & MCF-7 & FS4-LTM \\
\hline $\mathbf{1}$ & - & 68.8 & - & - \\
$\mathbf{2}$ & - & 25.8 & 77.5 & 32.2 \\
$\mathbf{4}$ & 94.3 & - & 78.6 & - \\
$\mathbf{5}$ & - & - & 206.9 & - \\
$\mathbf{6}$ & 117.6 & 88.2 & - & - \\
$\mathbf{7}$ & - & - & 60.0 & - \\
$\mathbf{8}$ & - & - & 47.2 & 87.3 \\
$\mathbf{1 0}$ & - & - & 90.6 & 73.4 \\
$\mathbf{1 5}$ & 55.9 & 48.9 & - & - \\
$\mathbf{1 6}$ & - & - & 96.3 & - \\
$\mathbf{1 7}$ & - & - & 64.8 & - \\
\hline
\end{tabular}

aCompounds with no activity are not listed in the table.

\section{Experimental}

UV spectra were recorded during HPLC separation with a DAD detector (JASCO MD-2010 Plus). CD spectra were recorded on a JASCO J-810 spectropolarimeter. Low and high resolution ESIMS was performed with a Bruker micrOTOF ${ }_{L C}$ mass spectrometer. Mass calibration was performed using sodium formate cluster ions prior each measurement. ${ }^{1} \mathrm{H}$ and ${ }^{13} \mathrm{C}$ spectra were recorded on a Bruker Avance $600 \mathrm{NMR}$ spectrometer equipped with a cryo platform $\left({ }^{1} \mathrm{H}\right.$ at $600 \mathrm{MHz},{ }^{13} \mathrm{C}$ at $\left.150 \mathrm{MHz}\right)$ and a Bruker Avance NMR spectrometer $\left({ }^{1} \mathrm{H}\right.$ at $400 \mathrm{MHz},{ }^{13} \mathrm{C}$ at $100 \mathrm{MHz}$ ). All NMR experiments were measured at a temperature of $303 \mathrm{~K}$ using DMSO- $d_{6}\left(\delta_{\mathrm{H}} 2.50, \delta_{\mathrm{C}} 39.5\right)$ as internal standard. HPLC separation was achieved by Jasco PU-1580 using a Kromasil RP18 column $(16 \mathrm{~mm} \times 250 \mathrm{~mm}, 5 \mu \mathrm{m})$ and a Kromasil RP18 column $(1 \mathrm{~mm} \times 50 \mathrm{~mm}, 5 \mu \mathrm{m})$ and was eluted with gradient $\mathrm{H}_{2} \mathrm{O}(0.1 \%$ TFA) and $\mathrm{MeCN}(0.1 \%$ TFA).

The sponge Aplysina lacunosa was collected by SCUBA diving at a depth of $8 \mathrm{~m}$ from Stirrup Cay in the Bahamas in June 2008. The sample was immediately frozen and kept at $-20^{\circ} \mathrm{C}$ until extraction. A voucher specimen of this species is deposited in AG Köck, Alfred-Wegener-Institut, Helmholtz-Zentrum für Polar- und Meeresforschung (voucher number: Aplysina lacunosa 08/21). The freeze-dried sponge (200 g) was extracted three times with $\mathrm{CH}_{2} \mathrm{Cl}_{2} / \mathrm{MeOH}(1: 1, \mathrm{v} / \mathrm{v})$ at room temperature. The filtrates were pooled and evaporated to yield $24.3 \mathrm{~g}$ of crude extract which was further partitioned between $n$-hexane and $\mathrm{MeOH}$. The $\mathrm{MeOH}$ extract was then partitioned between EtOAc and $\mathrm{H}_{2} \mathrm{O}$. The EtOAc fraction was further purified by vacuum liquid chromatography using silica gel eluting with stepwise gradient from 100:0 to 80:20 $\left(\mathrm{CH}_{2} \mathrm{Cl}_{2} / \mathrm{MeOH}\right.$, v/v). The fraction eluted with 97:3 $\left(\mathrm{CH}_{2} \mathrm{Cl}_{2} / \mathrm{MeOH}, \mathrm{v} / \mathrm{v}\right)$ was concentrated and further purified by HPLC using an RP C18 column (stepwise gradient 60:40, 40:60, and 20:80 $\mathrm{H}_{2} \mathrm{O} / \mathrm{MeCN}$, v/v) to 
yield 5 (198.0 mg), 11 (263.0 mg), 12 (445.0 mg), 13 (55.0 mg), 14 (7.9 mg), 16 (15.0 mg), 17 (9.1 mg), and two other fractions. The first fraction was purified using reversedphase HPLC [52:48 $\left.\mathrm{H}_{2} \mathrm{O}(0.1 \% \mathrm{TFA}) / \mathrm{MeCN}(0.1 \% \mathrm{TFA}), \mathrm{v} / \mathrm{v}\right]$ to obtain $7(3.2 \mathrm{mg}), \mathbf{8}(4.5 \mathrm{mg}), \mathbf{9}(9.6 \mathrm{mg})$ and following with analytical RP18 column [gradient $60: 40$ to $20: 80 \mathrm{H}_{2} \mathrm{O}$ $(0.1 \% \mathrm{TFA}) / \mathrm{MeCN}(0.1 \% \mathrm{TFA}), \mathrm{v} / \mathrm{v}]$ yielding $1(0.8 \mathrm{mg})$. The other fraction was purified using RP18 HPLC [60:40 $\mathrm{H}_{2} \mathrm{O}$ $(0.1 \% \mathrm{TFA}) / \mathrm{MeCN}(0.1 \% \mathrm{TFA}), \mathrm{v} / \mathrm{v}]$ and follow with an analytical RP18 column $\left[65: 35 \mathrm{H}_{2} \mathrm{O}(0.1 \%\right.$ TFA $) / \mathrm{MeCN}$ $(0.1 \% \mathrm{TFA}), \mathrm{v} / \mathrm{v}]$ yielding $2(0.8 \mathrm{mg})$ and $\mathbf{1 8}(5.3 \mathrm{mg})$. The 94:6 $\left(\mathrm{CH}_{2} \mathrm{Cl}_{2} / \mathrm{MeOH}, \mathrm{v} / \mathrm{v}\right)$ fraction was purified by HPLC using a RP C18 column (gradient 80:20 to $40: 60 \mathrm{H}_{2} \mathrm{O} / \mathrm{MeCN}, \mathrm{v} / \mathrm{v}$ ) yielding 6 (10.7 mg), $10(25.5 \mathrm{mg}), \mathbf{1 5}(5.0 \mathrm{mg})$, and one fraction which was purified using reversed-phase HPLC [70:30 $\mathrm{H}_{2} \mathrm{O}(0.1 \% \mathrm{TFA}) / \mathrm{MeCN}(0.1 \% \mathrm{TFA}), \mathrm{v} / \mathrm{v}$ ] to get a mixture of $\mathbf{3}$ and $\mathbf{1 5}(3.5 \mathrm{mg})$ as well as $\mathbf{4}(3.0 \mathrm{mg})$.

\section{Biological activity test}

The antimicrobial activities of isolated compounds were evaluated against five microorganisms [Gram-positive: Straptococcus aureus (MRSA and MSSA) and Micrococcus luteus; Gram-negative: Peumonia aruginosa and Klebsiella pneumonia] and antifungal Candida albicans using microdilution technique. The MIC was defined as lowest concentration that shows $50 \%$ growth inhibition after 24 hour incubation.

\section{Cytotoxicity assay}

The cytotoxicity was determined using WST-1 cell proliferation assays. Targeting cell lines are L929 mouse fibroblasts, KB-31 epidermoid carcinoma, and MCF-7 breast cancer cell lines which were incubated for 5 days with the test substances. The acute toxicity was determined using the FS4-LTM conditional immortalization human fibroblasts cell line which was incubated for 24 hours with the test compounds.

\section{Experimental data}

14-Debromo-11-deoxyfistularin-3 (1): white solid; UV (DAD) $\lambda_{\max } 226 \mathrm{~nm} ; \mathrm{CD}(\mathrm{MeOH}) \lambda_{\max } 248 \mathrm{~nm}(\Delta \varepsilon+5.16), 288 \mathrm{~nm}$ $(\Delta \varepsilon+4.55) ;{ }^{1} \mathrm{H}$ NMR and ${ }^{13} \mathrm{C}$ NMR see Table 1 ; HRMS-ESI $(+)$ $m / z=1036.7844[\mathrm{M}+\mathrm{Na}]^{+}$(calcd for $\mathrm{C}_{31} \mathrm{H}_{31}{ }^{79} \mathrm{Br}_{5} \mathrm{~N}_{4} \mathrm{O}_{10} \mathrm{Na}$, $1036.7855, \Delta m=1.1 \mathrm{ppm})$.

Aplysinin A (2): white solid; UV (DAD) $\lambda_{\max } 225 \mathrm{~nm}$; CD $(\mathrm{MeOH}) \lambda_{\max } 252 \mathrm{~nm}(\Delta \varepsilon+4.77), 283 \mathrm{~nm}(\Delta \varepsilon+3.34) ;{ }^{1} \mathrm{H}$ NMR and ${ }^{13} \mathrm{C}$ NMR see Table 3; HRMS-ESI $(+) \mathrm{m} / \mathrm{z}=793.8320$ $[\mathrm{M}+\mathrm{Na}]^{+}$(calcd for $\mathrm{C}_{23} \mathrm{H}_{25}{ }^{79} \mathrm{Br}_{4} \mathrm{~N}_{3} \mathrm{O}_{7} \mathrm{Na}, 793.8324$, $\Delta m=0.4 \mathrm{ppm})$.

Aplysinin B (3): white solid; UV (DAD) $\lambda_{\max } 227 \mathrm{~nm}$; ${ }^{1} \mathrm{H}$ NMR and ${ }^{13} \mathrm{C}$ NMR see Table 4; HRMS-ESI(+) $m / z=456.9892[\mathrm{M}+\mathrm{H}]^{+}\left(\right.$calcd for $\mathrm{C}_{16} \mathrm{H}_{19}{ }^{79} \mathrm{Br}_{2} \mathrm{~N}_{4} \mathrm{O}_{2}$, 456.9875, $\Delta m=1.7 \mathrm{ppm})$.

\section{Supporting Information}

\section{Supporting Information File 1}

1D, 2D NMR, and CD spectra of three new compounds.

1D NMR, mass and CD spetra of all known isolated compounds.

[http://www.beilstein-journals.org/bjoc/content/

supplementary/1860-5397-11-254-S1.pdf]

\section{Acknowledgements}

Financial support from the Deutsche Forschungsgemeinschaft (DFG) (Ko 1314/5-1 and 5-2, DFG-Forschergruppe FOR 934) is gratefully acknowledged. Sponge collection was carried out by Dr. Gesine Schmidt and Dr. Achim Grube during a scientific expedition to the Bahamas in 2008. We would like to acknowledge the support of Prof. Dr. Joseph R. Pawlik (University of North Carolina, Wilmington, USA) who gave members of the Köck research group the opportunity to participate in the research trips to the Bahamas. We further thank Dr. Sven Zea (Universidad Nacional de Colombia) for identification of the sponge samples, Dr. Florenz Sasse (Helmholtz Center for Infection Research, Braunschweig) for biological activity tests, and Dr. Andreas Hennig (Jacobs University, Bremen) for CD spectrometer access. The photograph in the graphical abstract was reproduced with permission from Sven Zea (http:// www.spongeguide.org).

\section{References}

1. Sharma, G. M.; Burkholder, P. R. Tetrahedron Lett. 1967, 8, 4147-4150. doi:10.1016/S0040-4039(01)89710-0

2. Gunasekera, S. P.; Cross, S. S. J. Nat. Prod. 1992, 55, 509-512. doi:10.1021/np50082a020

3. Kobayashi, J.; Tsuda, M.; Agemi, K.; Shigemori, H.; Ishibashi, M.; Sasaki, T.; Mikami, Y. Tetrahedron 1991, 47, 6617-6622. doi:10.1016/S0040-4020(01)82314-0

4. Andersen, R. J.; Faulkner, D. J. Tetrahedron Lett. 1973, 14, 1175-1178. doi:10.1016/S0040-4039(01)95788-0

5. Gao, H.; Kelly, M.; Hamann, M. T. Tetrahedron 1999, 55, 9717-9726. doi:10.1016/S0040-4020(99)00553-0

6. Okamoto, Y.; Ojika, M.; Kato, S.; Sakagami, Y. Tetrahedron 2000, 56, 5813-5818. doi:10.1016/S0040-4020(00)00544-5

7. Tsuda, M.; Shigemori, H.; Ishibashi, M.; Kobayashi, J. Tetrahedron Lett. 1992, 33, 2597-2598. doi:10.1016/S0040-4039(00)92253-6

8. Yagi, H.; Matsunaga, S.; Fusetani, N. Tetrahedron 1993, 49, 3749-3754. doi:10.1016/S0040-4020(01)90227-3

9. Ichiba, T.; Scheuer, P. J.; Kelly-Borges, M. J. Org. Chem. 1993, 58, 4149-4150. doi:10.1021/jo00067a062 
10. Ross, S. A.; Weete, J. D.; Schinazi, R. F.; Wirtz, S. S.; Tharnish, P.; Scheuer, P. J.; Hamann, M. T. J. Nat. Prod. 2000, 63, 501-503. doi:10.1021/np980414u

11. Tsukamoto, S.; Kato, H.; Hirota, H.; Fusetani, N. Tetrahedron 1996, 52 , 8181-8186. doi:10.1016/0040-4020(96)00387-0

12. Mierzwa, R.; King, A.; Conover, M. A.; Tozzi, S.; Puar, M. S.; Patel, M.; Coval, S. J.; Pomponi, S. A. J. Nat. Prod. 1994, 57, 175-177. doi:10.1021/np50103a029

13. Acosta, A. L.; Rodriguez, A. D. J. Nat. Prod. 1992, 55, 1007-1012. doi:10.1021/np50085a031

14. Tabudravu, J. N.; Jaspars, M. J. Nat. Prod. 2002, 65, 1798-1801. doi:10.1021/np020275n

15. Yang, X.; Davis, R. A.; Buchanan, M. S.; Duffy, S.; Avery, V. M.; Camp, D.; Quinn, R. J. J. Nat. Prod. 2010, 73, 985-987. doi:10.1021/np900834g

16. Galeano, E.; Martínez, A.; Thomas, O. P.; Robledo, S.; Munoz, D. Quim. Nova 2012, 35, 1189-1193. doi:10.1590/S0100-40422012000600023

17. Galeano, E.; Thomas, O. P.; Robledo, S.; Munoz, D.; Martinez, A. Mar. Drugs 2011, 9, 1902-1913. doi:10.3390/md9101902

18. James, D. M.; Kunze, H. B.; Faulkner, D. J. J. Nat. Prod. 1991, 54, 1137-1140. doi:10.1021/np50076a040

19. Gopichand, Y.; Schmitz, F. J. Tetrahedron Lett. 1979, 41, 3921-3924. doi:10.1016/S0040-4039(01)86465-0

20. Kernan, M. R.; Cambie, R. C.; Bérgquist, P. R. J. Nat. Prod. 1990, 53, 615-622. doi:10.1021/np50069a012

21. Mancini, I.; Guella, G.; Laboute, P.; Debitus, C.; Pietra, F. J. Chem. Soc., Perkin Trans. 1 1993, 3121-3125. doi:10.1039/p19930003121

22. Compagnone, R. S.; Avila, R.; Suárez, A. I.; Abrams, O. V.; Rangel, H. R.; Arvelo, F.; Piña, I. C.; Merentes, E. J. Nat. Prod. 1999, 62, 1443-1444. doi:10.1021/np9901938

23. Morris, S. A.; Andersen, R. J. Can. J. Chem. 1989, 67, 677-681. doi:10.1139/v89-102

24. Fattorusso, E.; Minale, L.; Sodano, G.; Moody, K.; Thomson, R. H. J. Chem. Soc. D 1970, 752-753. doi:10.1039/c29700000752

25. McMillan, J. A.; Paul, I. C.; Goo, Y. M.; Rinehart, K. L., Jr.; Krueger, W. C.; Pschigoda, L. M. Tetrahedron Lett. 1981, 22, 39-42. doi:10.1016/0040-4039(81)80035-4

26. Ciminiello, P.; Costantino, V.; Fattorusso, E.; Magno, S.; Mangoni, A. J. Nat. Prod. 1994, 57, 705-712. doi:10.1021/np50108a004

27. Assmann, M.; Wray, V.; van Soest, R. W. M.; Proksch, P. Z. Naturforsch., C 1998, 53, 398-401.

28. Minale, L.; Sodano, G.; Chan, W. R.; Chen, A. M. J. Chem. Soc., Chem. Commun. 1972, 674-675. doi:10.1039/c39720000674

29. Abou-Shoer, M. I.; Shaala, L. A.; Youssef, D. T. A.; Badr, J. M.; Habib, A.-A. M. J. Nat. Prod. 2008, 71, 1464-1467. doi:10.1021/np800142n

30. Nishiyama, S.; Yamamura, S. Bull. Chem. Soc. Jpn. 1985, 58, 3453-3456. doi:10.1246/bcsj.58.3453

31. Tilvi, S.; Rodrigues, C.; Naik, C. G.; Parameswaran, P. S.; Wahidhulla, S. Tetrahedron 2004, 60, 10207-10215. doi:10.1016/j.tet.2004.09.009

32. Köck, M.; Reif, B.; Fenical, W.; Griesinger, C. Tetrahedron Lett. 1996, 37, 363-366. doi:10.1016/0040-4039(95)02206-6

33. Fulmor, W.; van Lear, G. E.; Morton, G. O.; Mills, R. D. Tetrahedron Lett. 1970, 11, 4551-4552. doi:10.1016/S0040-4039(00)89414-9
34. Rogers, E. W.; de Oliveira, M. F.; Berlinck, R. G. S.; König, G. M.; Molinski, T. F. J. Nat. Prod. 2005, 68, 891-896. doi:10.1021/np050050n

35. Rogers, E. W.; Molinski, T. F. J. Nat. Prod. 2007, 70, 1191-1194. doi:10.1021/np070109|

36. Ebel, R.; Brenzinger, M.; Kunze, A.; Gross, H. J.; Proksch, P. J. Chem. Ecol. 1997, 23, 1451-1462. doi:10.1023/B:JOEC.0000006475.10310.3a

37. Ciminiello, P.; Dell'Aversano, C.; Fattorusso, E.; Magno, S.; Pansini, M. J. Nat. Prod. 1999, 62, 590-593. doi:10.1021/np9805138

38. Ciminiello, P.; Fattorusso, E.; Magno, S.; Pansini, M. J. Nat. Prod. 1994, 57, 1564-1569. doi:10.1021/np50113a016

\section{License and Terms}

This is an Open Access article under the terms of the Creative Commons Attribution License

(http://creativecommons.org/licenses/by/2.0), which permits unrestricted use, distribution, and reproduction in any medium, provided the original work is properly cited.

The license is subject to the Beilstein Journal of Organic Chemistry terms and conditions:

(http://www.beilstein-journals.org/bjoc)

The definitive version of this article is the electronic one which can be found at: doi:10.3762/bjoc. 11.254 